\title{
On the Decidability of Reachability in Linear Time-Invariant Systems
}

\author{
Nathanaël Fijalkow \\ CNRS, LaBRI, Bordeaux, France \\ Alan Turing Institute of Data Science, \\ London, United Kingdom \\ nathanael.fijalkow@labri.fr
}

\author{
Joël Ouaknine \\ Max Planck Institute for Software \\ Systems, Saarland Informatics \\ Campus, Germany \\ joel@mpi-sws.org
}

\author{
Amaury Pouly \\ CNRS, IRIF, Université Paris Diderot, \\ France \\ amaury.pouly@irif.fr
}

\author{
João Sousa-Pinto \\ Department of Computer Science, \\ University of Oxford, UK \\ jspinto@cs.ox.ac.uk
}

\author{
James Worrell \\ Department of Computer Science, \\ University of Oxford, UK \\ jbw@cs.ox.ac.uk
}

\begin{abstract}
We consider the decidability of state-to-state reachability in linear time-invariant control systems over discrete time. We analyse this problem with respect to the allowable control sets, which in general are assumed to be defined by boolean combinations of linear inequalities. Decidability of the version of the reachability problem in which control sets are affine subspaces of $\mathbb{R}^{n}$ is a fundamental result in control theory. Our first result is that reachability is undecidable if the set of controls is a finite union of affine subspaces. We also consider versions of the reachability problem in which (i) the set of controls consists of a single affine subspace together with the origin and (ii) the set of controls is a convex polytope. In these two cases we respectively show that the reachability problem is as hard as Skolem's Problem and the Positivity Problem for linear recurrence sequences (whose decidability has been open for several decades). Our main contribution is to show decidability of a version of the reachability problem in which control sets are convex polytopes, under certain spectral assumptions on the transition matrix.
\end{abstract}

\section{CCS CONCEPTS}

- Computing methodologies $\rightarrow$ Computational control theory;

\section{KEYWORDS}

LTI systems, control theory, reachability, decidability, recurrence sequences

\section{ACM Reference Format:}

Nathanaël Fijalkow, Joël Ouaknine, Amaury Pouly, João Sousa-Pinto, and James Worrell. 1997. On the Decidability of Reachability in Linear Time-Invariant Systems. In Proceedings of ACM Woodstock conference (WOODSTOCK'97). ACM, New York, NY, USA, 11 pages. https://doi.org/10.475/123_4

Permission to make digital or hard copies of part or all of this work for personal or classroom use is granted without fee provided that copies are not made or distributed for profit or commercial advantage and that copies bear this notice and the full citation on the first page. Copyrights for third-party components of this work must be honored.

For all other uses, contact the owner/author(s).

WOODSTOCK'97, fuly 1997, El Paso, Texas USA

(C) 2016 Copyright held by the owner/author(s).

ACM ISBN 123-4567-24-567/08/06

https://doi.org/10.475/123_4

\section{ACKNOWLEDGMENTS}

This project has received funding from the Alan Turing Institute under EPSRC grant EP/N510129/1. Joël Ouaknine was supported by ERC grant AVS-ISS (648701), and by the Deutsche Forschungsgemeinschaft (DFG, German Research Foundation) - Projektnummer 389792660 - TRR 248. Joël Ouaknine is also affiliated with the Department of Computer Science, University of Oxford, UK. Nathanaël Fijalkow and Amaury Pouly were supported by the CODYS project ANR-18-CE40-0007. James Worrell was supported by EPSRC Fellowship EP/N008197/1. Amaury Pouly did part of this work at the Max Planck Institute for Software Systems, Saarland Informatics Campus, Germany.

\section{INTRODUCTION}

This paper is concerned with linear time-invariant (LTI) systems. LTI systems are one of the most basic and fundamental models in control theory and have applications in circuit design, signal processing, and image processing, among many other areas. LTI systems have both discrete-time and continuous-time variants; here we are concerned solely with the discrete-time version.

A (discrete-time) LTI system in dimension $d$ is specified by a transition matrix $A \in \mathbb{Q}^{d \times d}$ and a set of controls $U \subseteq \mathbb{R}^{d}$. The evolution of the system is described by the recurrence $\boldsymbol{x}_{t+1}=A \boldsymbol{x}_{t}+\boldsymbol{u}_{t}$, where $\boldsymbol{u}_{t} \in U$ for all $t \in \mathbb{N}$. Here we think of the vectors $\boldsymbol{u}_{t}$ as inputs that are applied to the system.

Given such an LTI system, we say that state $s \in \mathbb{R}^{d}$ can reach state $t \in \mathbb{R}^{d}$ if there exists $T \geq 0$ and a sequence of controls $\boldsymbol{u}_{0}, \ldots, \boldsymbol{u}_{T-1} \in U$ such that the unique solution to the recurrence $\boldsymbol{x}_{t+1}=A \boldsymbol{x}_{t}+\boldsymbol{u}_{t}$ with initial condition $\boldsymbol{x}_{0}=\boldsymbol{s}$ satisfies $\boldsymbol{x}_{T}=\boldsymbol{t}$. The problem of computing the set of all states reachable from a given initial state has been an active topic of research for several decades. Here the emphasis is typically on efficient and scalable methods to over- and under-approximate the reachable set [8, 11, 12, 21, 33]. By contrast, relatively little attention has been paid to the decidability of the reachable set-the focus of the present paper. Specifically we consider the LTI Reachability Problem: given an LTI system, source state $\boldsymbol{s}$, and target state $\boldsymbol{t}$, decide whether $\boldsymbol{s}$ can reach $\boldsymbol{t}$. The main axis along which we delineate variants of the LTI Reachability Problem concerns the class of allowable control sets (e.g., affine subspaces, convex polytopes, etc.). 
Other reachability problems on LTI systems include so-called null reachability (can one reach all states from the origin?) and null controllability (can one reach the origin from all states?) [4]. However these "universal" reachability problems have a very different character to the point-to-point version that we study. In particular, both null reachability and null controllable are decidable in polynomial time using linear algebra.

One of the first people to address the LTI Reachability Problem was Harrison [15] who posed the question of whether the problem is decidable when the allowable control sets are vector subspaces of $\mathbb{R}^{d}$. (Harrison [15] called this the accessibility problem for linear sequential machines.) Harrison's question was resolved in a seminal paper of Lipton and Kannan [20], who gave a polynomial-time procedure for the LTI Reachability Problem in the case of linear control sets. The case in which the allowable control sets are affine subspaces of $\mathbb{R}^{d}$ (i.e., translates of linear subspaces) can easily be reduced to the linear case by a standard homogenisation trick.

The starting point of the present paper is to give a number of hardness results for relatively mild generalisations of Harrison's problem. Specifically we show that:

(1) If the allowable control sets are finite unions of affine subspaces of $\mathbb{R}^{d}$, then the LTI Reachability Problem is undecidable.

(2) If the allowable control sets are of the form $V \cup\{0\}$, with $V$ an affine subspace of $\mathbb{R}^{d}$, then the LTI Reachability Problem is as hard as Skolem's Problem for linear recurrence sequences.

(3) If the allowable control sets are convex polytopes then the LTI Reachability Problem is as hard as the Positivity Problem for linear recurrence sequences.

Skolem's Problem asks whether a given integer linear recurrence sequence has a zero term, while the Positivity Problem asks whether all terms of a given integer linear recurrence sequence are positive. The decidability of both problems has been open since the 1970s [14, 27, 28, 34]. To date, decidability of Skolem's Problem is known only for recurrences of order at most 4 [25,37] and decidability of the Positivity Problem is known only for recurrences of order at most 5 [26]. Thus the results in this paper suggest that deciding the LTI Reachability Problem for any class of control sets more general than affine subspaces will prove a very challenging problem. Note however that the problem is straightforwardly semidecidable, as reachability in $n$ steps for each fixed $n \in \mathbb{N}$ is easily reduced to solving a linear program.

Main Result. Our main result is a decision procedure for a version of the LTI Reachability Problem in which the initial state is the origin and the target is a convex polytope (generalising the case of reaching a single state). We assume that the control set is a convex polytopic neighbourhood of the origin. Intuitively, the condition that 0 lie in the interior of the control set ensures that we can control in every direction. As one might expect from the above discussion of hardness, our decision procedure requires fairly strong hypotheses on the transition matrix $A$ in order to work. Specifically we assume that (i) $A$ has spectral radius $\rho(A)<1$ and (ii) some positive power of $A$ has exclusively real spectrum (generalising the requirement that $A$ have real spectrum). Condition (i) is equivalent to the requirement that the system without input be asymptotically stable (also called Schur stable). Condition (ii) has appeared in closely related contexts, such as o-minimal hybrid systems (see [24, Theorem 6.2] and [30, Theorem 4.6]) and self-affine fractals (see below).

As we will show, without loss of generality we can restrict attention to LTI systems in which the set of vectors reachable from the origin is full dimensional. In this case, Condition (i) and the assumptions on the control set entail that the set of reachable vectors is a bounded convex open subset of $\mathbb{R}^{n}$. The essential challenge in deciding reachability is to handle the case in which the target point lies on the boundary of the reachable set. Condition (ii) plays two roles in this respect. First we use it to show that any unreachable point is separated from the reachable set by a hyperplane whose normal vector has algebraic-number coefficients (having previously observed that hyperplanes with rational normal vectors do not suffice: see Figure 3). Thus Condition (ii) ensures that we have an enumerable set of "witnesses" of non-reachability. Moreover we use this same condition to show that we can effectively verify such witnesses, i.e. decide whether some hyperplane with a given normal vector indeed separates the reachable set from the target point.

Related Work. It well understood that most control problems are undecidable for mild generalisations of linear systems $[5,6]$. For example, point-to-point reachability is undecidable for piecewise linear systems [3, 4, 23] and for saturated linear systems [31]. However to the best of our knowledge no previous work has attempted to systematically map the border of decidability for pointto-point reachability within the class of LTI systems. Indeed it is sometimes considered that point-to-point reachability is efficiently decidable for LTI systems (see, e.g., the discussion in [6, Section 4.1]). The results of this paper illustrate that the latter view crucially depends on the assumption that the set of controls $U$ form a linear (or affine) subspace. But such an assumption does not allow to express many natural requirements, e.g., that $U$ be bounded.

A range of different control problems for discrete- and continuoustime LTI systems under constraints on the set of controls have been studied in the literature $[1,9,11,13,16-19,29,32,33,35,38]$. Although it is very common to consider systems with saturated inputs, we do not consider the problem of controller design and thus saturated inputs reduce to having inputs in the unit hypercube in our case. LTI systems with convex input constraints have been considered in the past (see the references above) but we are not aware of any complete characterisation of the reachable set in this case, except in the case of conical constraints.

There is a clear relationship between the LTI Reachability Problem with bounded convex control sets and self-affine fractals. For LTI systems with spectral radius $\rho(A)<1$ and with input set $U$ a convex polytope, the closure of the set of states reachable from 0 is the convex hull of the self-affine fractal $\mathcal{F}$ arising as the unique solution of the set equation $\mathcal{F}=A \mathcal{F}+\operatorname{Ext}(U)$, where $\operatorname{Ext}(U)$ denotes the set of extreme points of $U$. We are aware of several results $[22,36]$ on the computability of the convex hulls of such fractals (and more general types of fractals). However those results only apply to the case when the convex hull is a polytope and usually only in dimension 2 . The requirements on the spectrum of $A$ in 
our positive decidability result are related to the so-called fractal of unity of [36].

\section{UNDECIDABILITY AND HARDNESS}

In this section we give evidence for the hardness of the LTI reachability problem. We show undecidability if the set of controls is a finite union of affine subspaces, we give a reduction from the Positivity Problem in case the set of controls is a bounded convex polytope, and we give a reduction from Skolem's Problem in case the set of controls is a union of two affine subspaces.

\subsection{Undecidability}

The goal of this subsection is to prove the following result.

THEOREM 2.1. The reachability problem for LTI systems whose sets of controls are finite unions of affine subspaces is undecidable.

We prove Theorem 2.1 by reduction from the vector reachability problem for invertible matrices: given invertible matrices $A_{1}, \ldots, A_{k} \in$ $\mathbb{Q}^{d \times d}$ and vectors $\boldsymbol{x}, \boldsymbol{y} \in \mathbb{Q}^{d}$, do there exist integers $n_{1}, \ldots, n_{k}$ such that $\prod_{i=1}^{k} A_{i}^{n_{i}} \boldsymbol{x}=\boldsymbol{y}$ ? The undecidability of this problem is folklore (but see [10] for a proof). The key idea underlying the reduction of this problem to the reachability problem for LTI systems is to form an LTI whose transition matrix $A$ incorporates $A_{1}, \ldots, A_{k}$, and to provide a set of controls that can be used to simulate the successive application of powers of $A_{1}, A_{2}$, etc, by repeated application of $A$ A subtle technical point here is to make the reduction robust with respect to the different orders in which the controls can be applied.

Proof of Theorem 2.1. We reduce the vector reachability problem for invertible matrices to the reachability problem for LTI systems.

Let $A_{1}, \ldots, A_{k} \in \mathbb{Q}^{d \times d}$ be invertible matrices and $\boldsymbol{x}, \boldsymbol{y} \in \mathbb{Q}^{d}$. From these data we define an LTI system $\mathcal{L}=(A, U)$ in dimension $D:=(k+1) d+k$. We consider the state space of $\mathcal{L}$ to be

$$
\underbrace{\mathbb{R}^{d} \oplus \cdots \oplus \mathbb{R}^{d}}_{k+1} \oplus \mathbb{R}^{k},
$$

that is, each state comprises a $(k+1)$-tuple of vectors in $\mathbb{R}^{d}$ followed by a single vector in $\mathbb{R}^{k}$.

Matrix $A$ is a block diagonal matrix of dimension $D \times D$, given by

$$
A:=\operatorname{diag}\left(I_{d}, A_{1}, \ldots, A_{k}, I_{k}\right) .
$$

For all $i \in\{1, \ldots, k\}$ define $V_{i} \subseteq \mathbb{R}^{D}$ by

$$
V_{i}:=\{\boldsymbol{0}\}^{i-1} \times\left\{(\boldsymbol{z},-\boldsymbol{z}): \boldsymbol{z} \in \mathbb{R}^{d}\right\} \times\{\mathbf{0}\}^{k-i} \times\left\{\boldsymbol{e}_{i}\right\},
$$

where $\boldsymbol{e}_{i} \in \mathbb{R}^{k}$ denotes $i$-th coordinate vector and $\mathbf{0}$ denotes the zero vector in $\mathbb{R}^{d}$.

We now define the set of controls $U \subseteq \mathbb{R}^{D}$ by

$$
U:=V_{1}+\cdots+V_{k} \text {. }
$$

Given $i \in\{1, \ldots, k\}$, we think of $V_{i}$ as being comprised of atomic controls. Such a control is determined by the index $i$ and a vector $z \in \mathbb{R}^{d}$. Application of the control subtracts $z$ from the $i$-th block within the global state and adds $z$ to the $(i+1)$-st block. Intuitively the definition of $U$ as a sum of the $V_{i}$ allows to apply several atomic controls at the same time. Note that $U$ can be written as a union of affine subspaces.

Finally, we define the initial state to be $s:=(x, 0, \ldots, 0,0)$ and the target state to be $\boldsymbol{t}:=(\mathbf{0}, \ldots, \mathbf{0 , y}, \mathbf{1})$.

This completes the definition of the LTI system. We now argue that $\boldsymbol{t}$ is reachable from $\boldsymbol{s}$ if and only if there exist $n_{1}, \ldots, n_{k} \in \mathbb{Z}$ such that $\prod_{i=1}^{k} A_{i}^{n_{i}} \boldsymbol{x}=\boldsymbol{y}$. We divide the argument into two claims.

Claim 2.2. Given integers $n_{1}, \ldots, n_{k}$, there exist non-negative integers $t_{1}, \ldots, t_{k+1}$ such that

$$
\begin{aligned}
n_{1} & =t_{2}-t_{1} \\
& \vdots \\
n_{k} & =t_{k+1}-t_{k} .
\end{aligned}
$$

Proof. The proof is by induction on $k$. The base case $(k=$ $1)$ is obvious. For the induction step, suppose we are given integers $n_{1}, \ldots, n_{k+1}$. By induction we can find nonnegative integers $t_{1}, \ldots, t_{k+1}$ such that (1) holds. We can moreover assume that $n_{k+1}+$ $t_{k+1} \geq 0$ since (1) will still hold if we translate all $t_{1}, \ldots, t_{k+1}$ by a common integer. Hence we can define $t_{k+2}:=n_{k+1}+t_{k+1}$ and we have $n_{k+1}=t_{k+2}-t_{k+1}$.

Claim 2.3. Vector $\boldsymbol{t}$ is reachable from $\boldsymbol{s}$ if and only if there exist nonnegative integers $t_{1}, \ldots, t_{k+1}$ and vectors $z_{1}, \ldots, z_{k+1} \in \mathbb{R}^{d}$ such that the following equations hold:

$$
\begin{aligned}
z_{1} & =\boldsymbol{x} \\
z_{i+1} & =A_{i}^{t_{i+1}-t_{i}} z_{i} \quad i \in\{1, \ldots, k\} \\
z_{k+1} & =\boldsymbol{y} .
\end{aligned}
$$

Proof. Suppose that $t$ is reachable from $s$. Note that the final $k$ coordinates of $s$ are all zero, while the corresponding coordinates of $t$ are all one. Since the block of matrix $A$ corresponding to these coordinates is the identity $I_{k}$, it follows that in going from $s$ to $t$ exactly one atomic control from each space $V_{i}$ was used for all $i \in$ $\{1, \ldots, k\}$. For each $i$ write $z_{i} \in \mathbb{R}^{d}$ for the vector that determines the control in $V_{i}$ and let $t_{i}$ denote the number of steps after which this control was applied. Finally define $z_{k+1}:=\boldsymbol{y}$ and let $t_{k+1}$ be the total number of steps going from $s$ to $t$. We will show that with these definitions (2) is satisfied.

Since the first block of $A$ is $I_{d}$, in order to reach a state of $\mathcal{L}$ whose first block is 0 we must have $z_{1}=\boldsymbol{x}$. In similar fashion, considering the $(i+1)$-st block for $i=1, \ldots, k$, we have

$$
A_{i}^{t_{k+1}-t_{i}} z_{i}-A_{i}^{t_{k+1}-t_{i+1}} z_{i+1}=0
$$

and hence, since $A_{i}$ is invertible, $z_{i+1}=A_{i}^{t_{i+1}-t_{i}} z_{i}$.

Conversely, suppose that there exist nonnegative integers $t_{1}, \ldots, t_{k+1}$ and vectors $z_{1}, \ldots, z_{k+1} \in \mathbb{R}^{d}$ satisfying (2). Then $\boldsymbol{t}$ is reachable from $s$ in $t_{k+1}$ steps by, for all $i \in\{1, \ldots, k\}$, applying at time $t_{i}$ the atomic control in $V_{i}$ that is determined by $z_{i}$. Indeed since we thereby apply one atomic control for each $i \in\{1, \ldots, k\}$ we reach a state with vector 1 in the final block. The equations in (2) moreover guarantee that the reached state has 0 in its first $k$ blocks and $\boldsymbol{y}$ in the $(k+1)$-st block, i.e., the reached state is identical to $\boldsymbol{t}$. 
We now complete the proof of Theorem 2.1 by combining the above two claims.

Suppose that there exist integers $n_{1}, \ldots, n_{k}$ such that $\prod_{i=1}^{k} A_{i}^{n_{i}} x=$ $\boldsymbol{y}$. By Claim 2.2 there exists nonnegative integers $t_{1}, \ldots, t_{k+1}$ satisfying (1). Then if we define $z_{i}:=\prod_{j=1}^{i-1} A^{n_{j}} \boldsymbol{x}$ for $i \in\{1, \ldots, k+1\}$ we have that Equation (2) is satisfied. By Claim 2.3 it follows that $t$ is reachable from $s$ in the LTI system $\mathcal{L}$.

Conversely suppose that $t$ is reachable from $s$. By Claim 2.3 the system (2) has a solution. Defining $n_{i}:=t_{i+1}-t_{i}$ for $i \in\{1, \ldots, k\}$ we have that $\prod_{i=1}^{k} A_{i}^{n_{i}} \boldsymbol{x}=\boldsymbol{y}$.

\subsection{Positivity Hardness for Convex Control Sets}

Consider a sequence of integers $\left\langle x_{n}: n \in \mathbb{N}\right\rangle$. We say that such a sequence satisfies a linear recurrence of orderd if there exist $a_{1}, \ldots, a_{d} \in$ $\mathbb{Z}$ such that

$$
x_{n}=\sum_{i=1}^{d} a_{i} x_{n-i}
$$

for all $n \geq d$. Skolem's Problem asks, given such a sequence (specified by a recurrence and its initial values $x_{0}, \ldots, x_{d-1}$ ), whether $x_{n}=0$ for some $n$. Likewise the Positivity Problem asks whether $x_{n} \geq 0$ for all $n$. Decidability of Skolem's Problem and the Positivity Problem has been open since the 1970s [14, 27, 28, 34]. To date, decidability of Skolem's Problem is known only for recurrences of order at most $4[25,37]$ and decidability of the Positivity Problem is known only for recurrences of order at most 5 [26]. There is a relatively straightforward reduction of Skolem's Problem to the Positivity Problem (which does not preserve the order of recurrences).

In this section we show that if the set of controls is a convex polytope then the LTI reachability problem is as hard as the Positivity Problem. Instead of reducing from the Positivity Problem directly, we give a reduction from the Markov Reachability Problem: given a column-stochastic matrix $M \in \mathbb{Q}^{d \times d}$, determine whether there exists $n \in \mathbb{N}$ such that $\left(M^{n}\right)_{1,2} \geq \frac{1}{2}$. A reduction from the Positivity Problem to the Markov Reachability Problem has been given in [2].

Theorem 2.4. There is a reduction from the Positivity Problem to the reachability problem for LTI systems whose sets of controls are compact convex polytopes (with rational vertices).

Proof. We give a polynomial-time reduction from the Markov Reachability Problem to the problem at hand. Given a column-stochastic matrix $M \in \mathbb{Q}^{d \times d}$, we define an LTI system comprising a matrix $A=\operatorname{diag}(M, 0,0,1) \in \mathbb{Q}^{(d+3) \times(d+3)}$ and a compact convex polytope

$U=\left\{(-x, y, z, z): x \geq 0,0 \leq y \leq x_{1}\right.$, and $\left.\sum_{i=1}^{d} x_{i}=z \leq 1\right\} \subseteq \mathbb{R}^{d+3}$.

The initial state is $\boldsymbol{s}=\left(\boldsymbol{e}_{2}, 0,0,0\right) \in \mathbb{Q}^{d+3}$ and target state $\boldsymbol{t}=$ $\left(0, \frac{1}{2}, 1,1\right) \in \mathbb{Q}^{d+3}$.

We argue that $t$ is reachable from $s$ if and only if there exists $n \in \mathbb{N}$ such that $\left(M^{n}\right)_{1,2} \geq \frac{1}{2}$.
First, suppose that there exists $n \in \mathbb{N}$ such that $\left(M^{n}\right)_{1,2} \geq \frac{1}{2}$. Consider the sequence of controls $\boldsymbol{u}_{0}=\cdots=\boldsymbol{u}_{n-2}=\mathbf{0}$ and $\boldsymbol{u}_{n-1}=$ $\left(-M^{n} \boldsymbol{e}_{2}, \frac{1}{2}, 1,1\right) \in U$. This sequence steers $\boldsymbol{s}$ to $\boldsymbol{t}$.

On the other hand, suppose that there exists a sequence of controls $\boldsymbol{u}_{0}, \ldots, \boldsymbol{u}_{n-1}$ controlling $\boldsymbol{s}$ to $\boldsymbol{t}$. Since the $(d+2)$-nd and $(d+3)$ rd coordinates of $\boldsymbol{t}$ are equal, noting that the matrix $A$ erases coordinate $d+2$ but not $d+3$, it follows that $\boldsymbol{u}_{n-1}$ is the only non-zero control, that is, $\boldsymbol{u}_{0}=\cdots=\boldsymbol{u}_{n-2}=\mathbf{0}$. Therefore at time $n-1$ the state is $\left(M^{n-1} \boldsymbol{e}_{2}, 0,0,0\right)$, and the only way to reach $\boldsymbol{t}$ in the remaining step is to take $\boldsymbol{u}_{n-1}=\left(-M^{n-1} \boldsymbol{e}_{2}, \frac{1}{2}, 1,1\right) \in U$ (otherwise one of the first $d$ coordinates will be non-zero). But this is only possible if $\left(M^{n}\right)_{1,2} \geq \frac{1}{2}$.

To conclude the proof, observe that the LTI reachability instance $(\boldsymbol{s}, A, U, t)$ can be constructed in polynomial time from $M$.

\subsection{Skolem Hardness}

In this section we consider the case that the set of controls is the union of an affine subspace and the origin. We show that in this case the LTI reachability problem is as hard as Skolem's Problem.

We will work with the following matricial version of Skolem's Problem, rather than the formulation in terms of linear recurrences. The two versions are easily seen to be interreducible [27].

Definition 2.5. Given a matrix $M \in \mathbb{Q}^{d \times d}$, Skolem's problem consists in determining whether there exists a number $n \in \mathbb{N}$ such that $\left(M^{n}\right)_{1,2}=0$.

We will now show the following result:

THEOREM 2.6. There is a reduction from Skolem's Problem to the reachability problem for LTI systems whose set of controls is the unions of an affine subspace (with a basis of rational vectors) and the origin.

Proof. We give a (polynomial-time) reduction from the Skolem Problem to the problem at hand. Given a matrix $M \in \mathbb{Q}^{d \times d}$ we define the matrix $A=\operatorname{diag}(M, 2) \in \mathbb{Q}^{(d+1) \times(d+1)}$ and the set of admissible controls $\mathcal{P}=\{0\} \cup\left\{\left(0, x_{2}, \ldots, x_{d}, 1\right) \mid x_{2}, \ldots, x_{d} \in \mathbb{R}\right\}$, as well as the source $s=\left(\boldsymbol{e}_{2}, 0\right) \in \mathbb{Q}^{d+1}$ and target $\boldsymbol{t}=(0, \ldots, 0,1) \in$ $\mathbb{Q}^{d+1}$.

We argue that that $t$ is reachable from $s$ if and only if there exists $n \in \mathbb{N}$ such that $\left(M^{n}\right)_{1,2}=0$.

First, suppose that there exists $n \in \mathbb{N}$ such that $\left(M^{n}\right)_{1,2}=0$. Consider the sequence of controls given by $\boldsymbol{u}_{0}=\cdots=\boldsymbol{u}_{n-2}=\mathbf{0}$ and

$$
\boldsymbol{u}_{n-1}=\left(0,-\left(M^{n}\right)_{2,1},-\left(M^{n}\right)_{3,1}, \ldots,-\left(M^{n}\right)_{n, 1}, 1\right) .
$$

This sequence steers $\boldsymbol{s}$ to $\boldsymbol{t}$.

On the other hand, suppose there exists a sequence of controls $\boldsymbol{u}_{0}, \ldots, \boldsymbol{u}_{n-1}$ steering $\boldsymbol{s}$ to $\boldsymbol{t}$. The very last dimension implies that a vector in the second set of controls can be played only once, and in the last step. So the sequence of controls is zero vectors except at the last step. Looking at the first coordinate this implies that $\left(M^{n}\right)_{1,2}=0$.

\section{DECIDABILITY OF REACHABILITY FOR SIMPLE LTI SYSTEMS}

Define an LTI system $\mathcal{L}=(A, U)$ to be simple if: 
(1) the set of controls $U \subseteq \mathbb{R}^{d}$ is a bounded convex polytope that contains 0 in its relative interior (i.e. $0 \in B \cap \operatorname{Span}(U) \subseteq$ $U$ for some open ball $B$ around $\mathbf{0}$ );

(2) the spectral radius of $A$ is less than one;

(3) some positive power of $A$ has exclusively real spectrum

The rationale behind those assumptions will be explained in the following paragraphs.

We show decidability of the following problem: given a simple LTI system in dimension $d$ and a bounded convex polytope $Q \subseteq \mathbb{R}^{d}$, determine whether $Q$ is reachable from 0 . We call this the reachability problem for simple LTI systems. The set of states reachable from 0 is $A^{*}(U):=\bigcup_{m=0}^{\infty} \sum_{i=0}^{m} A^{i}(U)$. Thus the reachability problem for simple LTI systems is equivalent to asking whether $A^{*}(U)$ meets $Q$.

It is clear that the reachability problem for LTI systems is semidecidable. Fixing $n \in \mathbb{N}$, the problem of whether $\sum_{i=0}^{n} A^{i}(U)$ meets a given polytope $Q$ can straightforwardly be cast as a linear program. Iterating over all $n \in \mathbb{N}$ we thus have a semi-decision procedure for reachability.

In the rest of this section we describe a semi-decision procedure for non-reachability. The idea is to use a hyperplane that separates $A^{*}(U)$ and $Q$ as a certificate of non-reachability, as illustrated in Figure 1. The key technical step here is to show that it suffices to consider hyperplanes whose normal vectors have algebraic entries. To establish this we consider the cone of all hyperplanes that separate $A^{*}(U)$ and $Q$ and show that the extremal elements of this cone are algebraic. This reasoning heavily relies on the spectral assumptions about the matrix $A$ in the definition of simple LTI systems.

The main difficulty in certifying non-reachability is that the convex set $A^{*}(U)$ is difficult to describe in general. In particular, it can have infinitely many faces, even though the control polyhedron only has finitely many faces, as illustrated in Figure 2 . This is, however, not the only difficulty. Indeed, one might get the impression from Figure 2 that the boundary of $A^{*}(U)$ consists solely of (possibly countably many) facets, that is faces of dimension $d-1$. If that were true then one could one could separate $A^{*}(U)$ from any point not in $A^{*}(U)$ by a hyperplane that supports one of the facets of $A^{*}(U)$. Moreover since for a simple LTI system any facet of $A^{*}(U)$ has a supporting hyperplane with a rational normal vector, this would mean that for points outside $A^{*}(U)$ one can always find separating hyperplanes with rational coefficients. Unfortunately however there are cases, as illustrated in Figure 3, where part of the boundary of the reachable set does not belong to any facet, but rather to some lower-dimensional face. Such faces, by definition, do not usually have a unique supporting hyperplane and it is not a priori clear that they admit a supporting hyperplane with algebraic coefficients. The main technical result of this section (Proposition 3.9) shows that such algebraic supporting hyperplanes can always be found. It follows that for any point not in $A^{*}(U)$ there is always a separating hyperplane whose normal has algebraicnumber coefficients.

The following proposition identifies some simplifying assumptions that can be made without loss of generality for analysing reachability.

Proposition 3.1. The reachability problem for a simple LTI system $\mathcal{L}=(A, U)$ can be reduced to the special case in which it is assumed that all eigenvalues of $A$ are real and strictly positive and that $A^{*}(U)$ is full dimensional.

Proof. We first reduce to the case in which all eigenvalues of $A$ are real and nonnegative. Let $M$ be the least common multiple of the orders ${ }^{1}$ of the roots of unity $\frac{\lambda}{|\lambda|}$ for $\lambda$ a non-zero eigenvalue of $A$. Then $A^{M}$ has spectrum included in the nonnegative real line. But $Q \subseteq \mathbb{R}^{d}$ is reachable in the original LTI iff it is reachable in the LTI with transition matrix $A^{M}$ and set of controls $\sum_{i=0}^{M-1} A^{i}(U)$.

We next give a reduction to the case that $A$ is invertible. Write $\mathbb{R}^{d}=V_{0} \oplus V_{1}$, where $V_{0}, V_{1} \subseteq \mathbb{R}^{d}$ are $A$-invariant subspaces such that $A$ is nilpotent on $V_{0}$ (of index at most $d$ ) and invertible on $V_{1}$. Then for all $n \geq d$ and $\boldsymbol{u}_{0}, \ldots, \boldsymbol{u}_{n} \in U$ we have

$$
\sum_{i=0}^{n} A^{i} \boldsymbol{u}_{i}=\sum_{i=0}^{d-1} A^{i} \boldsymbol{u}_{i}+\sum_{i=0}^{n-d} A^{i} A^{d} \boldsymbol{u}_{i+d} .
$$

Suppose that $V_{1}$ has dimension $d_{1}$. Pick a basis of $V_{1}$ and with respect to this basis let $U^{\prime} \subseteq \mathbb{R}^{d_{1}}$ represent the set $A^{d}(U) \subseteq V_{1}$, let $Q^{\prime} \subseteq \mathbb{R}^{d_{1}}$ represent $\left(Q-\sum_{i=0}^{d-1} A^{i}(U)\right) \cap V_{1}$, and let $A^{\prime}$ represent the linear transformation on $V_{1}$ induced by $A$. From (3) it is clear that $Q$ is reachable in $\mathcal{L}$ if and only if $Q^{\prime}$ is reachable in the LTI system $\mathcal{L}^{\prime}:=\left(A^{\prime}, U^{\prime}\right)$, in which $A^{\prime}$ is an invertible matrix.

Now suppose that $\mathcal{L}=(A, U)$ a simple LTI system in dimension $d$ with $A$ invertible. Let $V \subseteq \mathbb{R}^{d}$ be the least $A$-invariant subspace that contains $U$. Then restricting $A$ to $V$ one obtains an LTI system in which $A^{*}(U)$ is full dimensional (with the restriction of $A$ to $V$ remaining invertible).

In the rest of this section we will assume that all simple LTI systems are such that $A^{*}(U)$ is full dimensional and all eigenvalues of $A$ are strictly positive.

Proposition 3.2. Given a simple LTI system $\mathcal{L}=(A, U)$, the reachable set $A^{*}(U)$ is a convex open subset of $\mathbb{R}^{d}$ whose closure is $A^{\infty}(U):=\sum_{i=0}^{\infty} A^{i}(U)$.

Proof. Since $\mathbf{0} \in U$ it holds that

$$
A^{0}(U) \subseteq A^{0}(U)+A^{1}(U) \subseteq A^{0}(U)+A^{1}(U)+A^{2}(U) \subseteq \cdots
$$

is an increasing chain of convex subsets of $\mathbb{R}^{d}$ and hence the union of the chain $A^{*}(U)$ is convex.

We next show that $A^{*}(U)$ is open. Now the increasing sequence of vector spaces $V_{0} \subseteq V_{1} \subseteq \ldots$, where $V_{j}:=\operatorname{Span}\left(\sum_{i=0}^{j} A^{i}(U)\right)$, stabilises in at most $d$ steps. Thus from the assumption that $A^{*}(U)$ is full dimensional we conclude that $\sum_{i=0}^{d-1} A^{i}(U)$ is already full dimensional. Furthermore, by Assumption 1 in the definition of Simple LTI system, we in fact have that $\sum_{i=0}^{d-1} A^{i}(U)$ contains a full-dimensional subset that is symmetric around 0 and hence contains $\mathbf{0}$ in its interior. Now consider a typical element $\sum_{i=0}^{n} A^{i} \boldsymbol{u}_{i} \in$ $A^{*}(U)$. Since $A$ is invertible we have that $A^{n+1}\left(\sum_{i=0}^{d-1} A^{i}(U)\right)=$ $\sum_{i=n+1}^{d+n} A^{i}(U)$ is also full dimensional and contains $\mathbf{0}$ in its interior. We conclude that $\sum_{i=0}^{n} A^{i} \boldsymbol{u}_{i}$ lies in the interior of $\sum_{i=0}^{n} A^{i} \boldsymbol{u}_{i}+$ $\sum_{i=n+1}^{d+n} A^{i}(U)$, which itself is contained in the interior of $A^{*}(U)$.

\footnotetext{
${ }^{1} \mathrm{~A}$ root of unity of order $r$ has degree $\varphi(r) \geq \frac{r}{283 \log \log r}$ as an algebraic number, where $\varphi(r)$ is Euler's function (see, e.g., [14]). From this fact it is straightforward to compute an upper bound on $M$.
} 

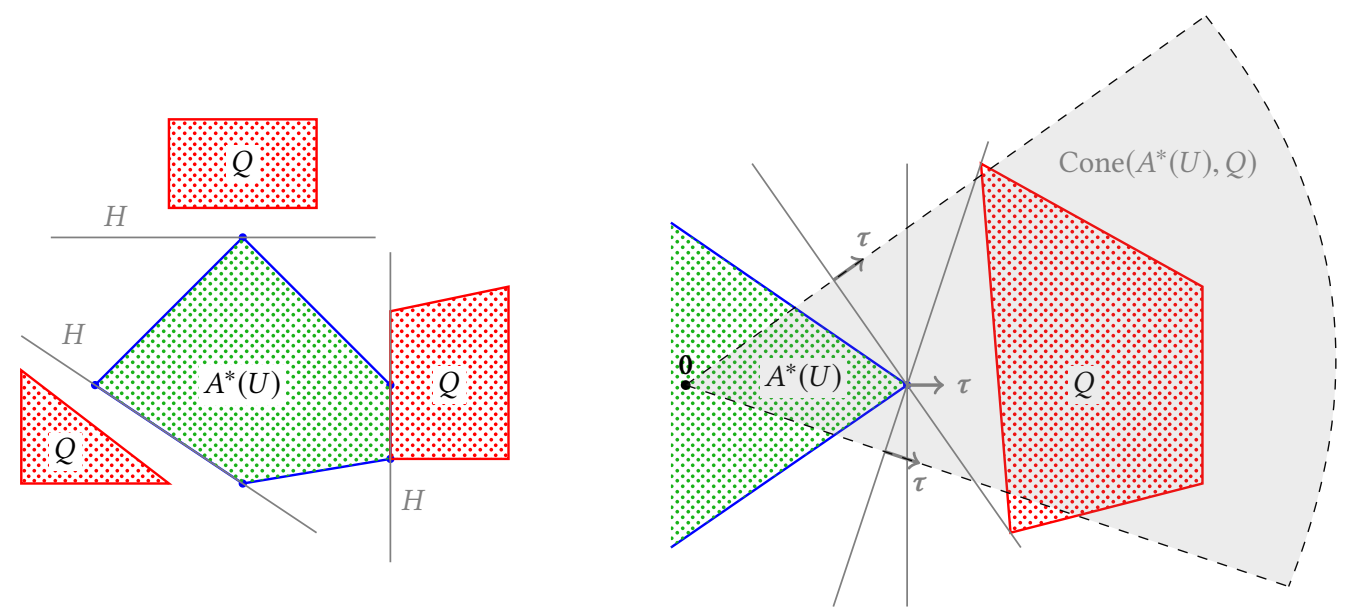

Figure 1: Example of separating hyperplanes $H$ between the reachable set $A^{*}(U)$ and various targets polytopes $Q$. If $Q$ and $A^{*}(U)$ are disjoint, we can always separate them by a supporting hyperplane of $A^{\infty}(U)$, the closure of $A^{*}(U)$. The set Cone $\left(A^{*}(U), Q\right)$ of all separators is crucial to understanding the situation. Note that the direction of such a supporting hyperplane may not always correspond to a facet of $A^{\infty}(U)$ or $Q$, in particular see Figure 3.

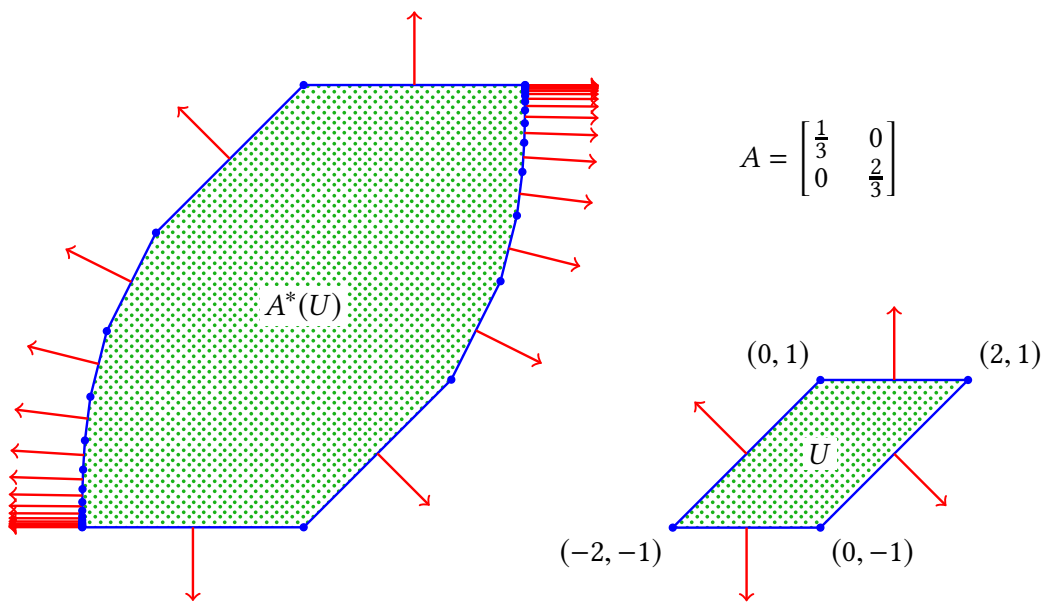

Figure 2: Example of a simple LTI system where the reachable set $A^{*}(U)$ is a convex set with infinitely many faces. Note that since $A^{*}(U)$ is open, we represented its closure $A^{\infty}(U)$.

From the fact that the spectral radius of $A$ is strictly less than one it easily follows that $A^{*}(U)$ is dense in $A^{\infty}(U)$, so it remains to observe that $A^{\infty}(U)$ is closed. But $A^{\infty}(U)$ is a fixed point of the contractive self map $F: X \mapsto A(X)+U$ on the metric space of all bounded subsets of $\mathbb{R}^{d}$ under the Hausdorff metric. Such a selfmap has a unique fixed point. Moreover, since the collection of compact subsets of $\mathbb{R}^{d}$ is complete under the Hausdorff metric and is preserved by $F$, we conclude that $A^{\infty}(U)$ is compact and thus closed.

The following is a (version of a) classical result of convex analysis:

Theorem 3.3 (Theorem of the Separating Hyperplane). Let $C$ and $D$ be compact convex subsets of $\mathbb{R}^{d}$. Then $\operatorname{int}(C) \cap D=\emptyset$ if and only if there exists $\tau \in \mathbb{R}^{d}$ and $b \in \mathbb{R}$ such that $\langle\boldsymbol{x}, \boldsymbol{\tau}\rangle \leq b$ for all $x \in C$ and $\langle\boldsymbol{x}, \boldsymbol{\tau}\rangle \geq \boldsymbol{b}$ for all $\boldsymbol{x} \in D$.

It follows from Proposition 3.2 and Theorem 3.3 that $A^{*}(U)$ and $Q$ are disjoint if and only if there exists $\tau \in \mathbb{R}^{d}$ and $b \in \mathbb{R}$ such that $\langle\boldsymbol{x}, \boldsymbol{\tau}\rangle \leq b$ for all $\boldsymbol{x} \in A^{\infty}(U)$ and $\langle\boldsymbol{x}, \boldsymbol{\tau}\rangle \geq b$ for all $\boldsymbol{x} \in Q$. This motivates us to define the cone of separators of $A^{*}(U)$ and $Q$ to be

Cone $\left(A^{\infty}(U), Q\right):=\left\{\boldsymbol{\tau} \in \mathbb{R}^{d}: \forall \boldsymbol{u} \in A^{\infty}(U) \forall \boldsymbol{v} \in Q,\langle\boldsymbol{u}, \boldsymbol{\tau}\rangle \leq\langle\boldsymbol{v}, \boldsymbol{\tau}\rangle\right\}$.

It is straightforward to verify that $\operatorname{Cone}\left(A^{\infty}(U), Q\right)$ is a topologically closed cone in $\mathbb{R}^{d}$. Moreover by the assumption that $A^{\infty}(U)$ is full dimensional we have that $\operatorname{Cone}\left(A^{\infty}(U), Q\right)$ is a pointed cone, that is, for all $\boldsymbol{\tau} \in \mathbb{R}^{d}$ if both $\boldsymbol{\tau},-\boldsymbol{\tau} \in \operatorname{Cone}\left(A^{\infty}(U), Q\right)$ then $\boldsymbol{\tau}=\mathbf{0}$. See Figure 1 for graphical representation of the cone. 

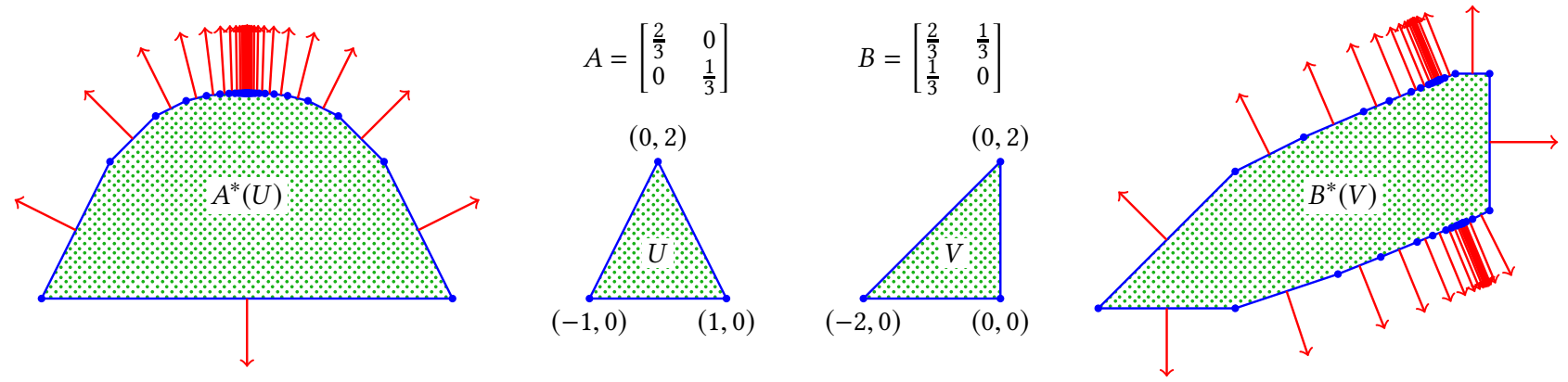

Figure 3: Example of simple LTI system in which an extreme point does not belong to a facet: the point $x=(0,3)$ "at the top" of $A^{*}(U)$, on the left, does not belong to any of the infinitely many edges that "converge" to it. In particular $x$ is extreme and exposed but it is not a vertex. It can be separated from $A^{*}(U)$ by the hyperplane $y \geqslant 3$ which has rational coefficients. However this is not always the case: $B^{*}(V)$ has two extreme points that do not belong to any facet and have rational coordinates, but whose (unique) separating hyperplane requires the use of algebraic irrationals. In particular, the normal to the separating hyperplanes is, up to sign, one the eigenvectors of $B$.

The main goal of Propositions 3.5-3.9 is to show the following lemma. Intuitively, if the boundary of the reachable set is a face, then there is only one possible direction for a separator (the normal to the face) and it is algebraic because the face has an algebraic description (Proposition 3.5). If it is a lower dimensional face, then there is a cone of possible directions and we need to show that it contains an algebraic one. The idea is to choose a vector satisfying particular conditions in the hope that those conditions allow us to recover unicity of the direction (and algebraicity by writing equations with algebraic entries). One such condition is to consider an extremal vector $\tau$ of the cone. Intuitively, a vector is extremal because of two possible reasons: either because the supporting hyperplane is "blocked" by $Q$ (see Figure 1), then we get (algebraic) equations from $Q$. Or because changing $\tau$ would change the face that the hyperplane is supporting (see Figure 4), then we get that $\tau$ is "dominating" in some sense, and this gives us further equations. The crux of of the proof is Proposition 3.9 showing that when all factors are considered, there is essentially a unique separating direction and therefore it must be algebraic.

LEMMA 3.4. If there is a separator of $A^{*}(U)$ and $Q$ then there is an algebraic separator.

Given $\tau \in \mathbb{R}^{d}$ and a closed set $C \subseteq \mathbb{R}^{d}$, define

$$
\begin{aligned}
\max _{\boldsymbol{\tau}}(C):= & \{\boldsymbol{v} \in C: \forall \boldsymbol{w} \in C,\langle\boldsymbol{v}, \boldsymbol{\tau}\rangle \geq\langle\boldsymbol{w}, \boldsymbol{\tau}\rangle\} \\
\min _{\boldsymbol{\tau}}(C):= & \{\boldsymbol{v} \in C: \forall \boldsymbol{w} \in C,\langle\boldsymbol{v}, \boldsymbol{\tau}\rangle \leq\langle\boldsymbol{w}, \boldsymbol{\tau}\rangle\} .
\end{aligned}
$$

For fixed $\boldsymbol{\tau} \in \mathbb{R}^{d}$, note that $\left\langle\sum_{i=0}^{\infty} A^{i} \boldsymbol{u}_{i}, \boldsymbol{\tau}\right\rangle$ is maximised for $\boldsymbol{u}_{0}, \boldsymbol{u}_{1}, \ldots \in U$ if and only if each individual inner product $\left\langle A^{i} \boldsymbol{u}_{i}, \boldsymbol{\tau}\right\rangle$ is maximised. In other words,

$\max _{\boldsymbol{\tau}}\left(A^{\infty}(U)\right)=\max _{\boldsymbol{\tau}}\left(A^{0}(U)\right)+\max _{\boldsymbol{\tau}}\left(A^{1}(U)\right)+\max _{\boldsymbol{\tau}}\left(A^{2}(U)\right)+\cdots$

Given $S \subseteq \mathbb{R}^{d}$, define

$$
\operatorname{Aff}_{0}(S)=\left\{\sum_{i=1}^{m} \lambda_{i} \boldsymbol{u}_{i}: \sum_{i=1}^{m} \lambda_{i}=0 \text { and } \boldsymbol{u}_{1}, \ldots, \boldsymbol{u}_{m} \in S, m \in \mathbb{N}\right\}
$$

Then $\operatorname{Aff}_{0}(S)$ is a vector subspace of $\mathbb{R}^{d}$-indeed $\operatorname{Aff}_{0}(S)$ is the unique translation of the affine hull of $S$ that contains the origin.

Proposition 3.5. The vector space $\mathrm{Aff}_{0}\left(\max _{\tau}\left(A^{\infty}(U)\right)\right)$ has a basis of rational vectors and $\mathrm{Aff}_{0}\left(\max _{\tau}\left(A^{i}(U)\right)\right) \subseteq \operatorname{Aff}_{0}\left(\max _{\tau}\left(A^{\infty}(U)\right)\right)$ for any $i \in \mathbb{N}$.

Proof. Let $n \in \mathbb{N}$ be such that

$\operatorname{Aff}_{0}\left(\max _{\tau}\left(A^{i}(U)\right)\right) \subseteq \operatorname{Aff}_{0}\left(\max _{\tau}\left(A^{0}(U)\right)\right)+\cdots+\operatorname{Aff}_{0}\left(\max _{\tau}\left(A^{n}(U)\right)\right)$

for all $i \in \mathbb{N}$. Such an $n$ exists since the right-hand side of (5) forms an increasing family of subspaces of $\mathbb{R}^{d}$ as $n \rightarrow \infty$ and such a sequence must eventually stabilize. We claim that

$\operatorname{Aff}_{0}\left(\max _{\tau}\left(A^{\infty}(U)\right)\right)=\operatorname{Aff}_{0}\left(\max _{\tau}\left(A^{0}(U)\right)\right)+\cdots+\operatorname{Aff}_{0}\left(\max _{\tau}\left(A^{n}(U)\right)\right)$.

From the claim it follows that $\operatorname{Aff}_{0}\left(\max _{\tau}\left(A^{\infty}(U)\right)\right)$ has a basis of rational vectors since $\max _{\tau}\left(A^{i}(U)\right)$ is bounded polytope with rational vertices for $i=0, \ldots, n$.

It remains to prove the claimed equality (6). The left-to-right inclusion follows directly from Equations (4) and (5). For the rightto-left inclusion, it suffices to note that for all $i \in \mathbb{N}$ we have that $\operatorname{Aff}_{0}\left(\max _{\tau}\left(A^{i}(U)\right)\right) \subseteq \operatorname{Aff}_{0}\left(\max _{\tau}\left(A^{\infty}(U)\right)\right)$. Indeed, suppose that $A^{i} \boldsymbol{u}, A^{i} \boldsymbol{v} \in \max _{\boldsymbol{\tau}}\left(A^{i}(U)\right)$ for some $\boldsymbol{u}, \boldsymbol{v} \in U$ and $i \in \mathbb{N}$. Define $\boldsymbol{u}_{j}, \boldsymbol{v}_{j} \in U$ for $j \in \mathbb{N}$ by $\boldsymbol{u}_{i}=\boldsymbol{u}, \boldsymbol{v}_{i}=\boldsymbol{v}$, and $A^{j} \boldsymbol{u}_{j}=A^{j} \boldsymbol{v}_{j} \in$ $\max _{\tau}\left(A^{j}(U)\right)$ for $j \neq i$. Then

$$
A^{i} \boldsymbol{u}-A^{i} \boldsymbol{v}=\sum_{j=0}^{\infty} A^{j} \boldsymbol{u}_{j}-\sum_{j=0}^{\infty} A^{j} \boldsymbol{v}_{j} \in \operatorname{Aff}_{0}\left(\max _{\tau}\left(A^{\infty}(U)\right)\right) .
$$

Proposition 3.6. Let the eigenvalues of matrix $A \in \mathbb{Q}^{d \times d}$ be $0<\lambda_{1}<\ldots<\lambda_{k}$. Then there is a collection of bilinear forms $L_{i j}: \mathbb{R}^{d} \times \mathbb{R}^{d} \rightarrow \mathbb{R}$ with algebraic coefficients such that for all $\boldsymbol{u}, \boldsymbol{\tau} \in \mathbb{R}^{d}$ we have

$$
\left\langle A^{n} \boldsymbol{u}, \boldsymbol{\tau}\right\rangle=\sum_{i=1}^{k} \sum_{j=0}^{d-1}\left(\begin{array}{l}
n \\
j
\end{array}\right) \lambda_{i}^{n} L_{i j}(\boldsymbol{u}, \boldsymbol{\tau}) .
$$


Proof. By the Jordan-Chevalley decomposition, we have $A=$ $P^{-1} D P+N$ where $D$ is diagonal, $N$ is nilpotent, $P^{-1} D P$ and $N$ commute, and all matrices have algebraic coefficients and can be computed $^{2}$. Moreover we can write $D=\lambda_{1} D_{1}+\cdots+\lambda_{k} D_{k}$ for appropriate idempotent diagonal matrices $D_{1}, \ldots, D_{k}$. Then for all $n \in \mathbb{N}$ we have

$$
\begin{aligned}
\left\langle A^{n} \boldsymbol{u}, \boldsymbol{\tau}\right\rangle & =\boldsymbol{\tau}^{\top}\left(P^{-1} D P+N\right)^{n} \boldsymbol{u} \\
& =\boldsymbol{\tau}^{\top} \sum_{j=0}^{n}\left(\begin{array}{l}
n \\
j
\end{array}\right) P^{-1} D^{n-j} P N^{j} \boldsymbol{u} \\
& =\boldsymbol{\tau}^{\top} \sum_{j=0}^{n}\left(\begin{array}{l}
n \\
j
\end{array}\right) P^{-1}\left(\lambda_{1}^{n-j} D_{1}+\cdots+\lambda_{k}^{n-j} D_{k}\right) P N^{j} \boldsymbol{u} \\
& =\sum_{i=1}^{k} \lambda_{i}^{n} \sum_{j=0}^{d-1}\left(\begin{array}{l}
n \\
j
\end{array}\right) \underbrace{\lambda_{i}^{-j} \boldsymbol{\tau}^{\top} P^{-1} D_{i} P N^{j} \boldsymbol{u}}_{=: L_{i j}(\boldsymbol{u}, \boldsymbol{\tau})} \\
& =\sum_{i=1}^{k} \sum_{j=0}^{d-1}\left(\begin{array}{l}
n \\
j
\end{array}\right) \lambda_{i}^{n} L_{i j}(\boldsymbol{u}, \boldsymbol{\tau}),
\end{aligned}
$$

where $L_{i j}(\boldsymbol{u}, \boldsymbol{\tau})$ is defined in (8). This concludes the proof because each $L_{i, j}$ is clearly bilinear with algebraic coefficients.

Given $\tau \in \mathbb{R}^{d}$, define $\operatorname{Dom}_{\mathcal{L}}(\tau)$ to be the linear subspace of $\mathbb{R}^{d}$ comprising all $\boldsymbol{\tau}^{\prime}$ such that for all $\boldsymbol{u}, \boldsymbol{u}^{\prime} \in \operatorname{Ext}(U)$ and every bilinear form $L_{i j}$ as in (7) if $L_{i j}\left(\boldsymbol{u}-\boldsymbol{u}^{\prime}, \boldsymbol{\tau}\right)=0$ then $L_{i j}\left(\boldsymbol{u}-\boldsymbol{u}^{\prime}, \boldsymbol{\tau}^{\prime}\right)=0$. It is clear that $\operatorname{Dom}_{\mathcal{L}}(\tau)$ that has a basis of vectors all of whose entries are algebraic numbers. Indeed, $\boldsymbol{\tau}^{\prime} \in \operatorname{Dom}_{\mathcal{L}}(\boldsymbol{\tau})$ if it satisfies some equations of the form $L_{i j}\left(\boldsymbol{u}-\boldsymbol{u}^{\prime}, \boldsymbol{\tau}^{\prime}\right)=0$ but $L_{i j}$ is bilinear, has algebraic coefficients and $\boldsymbol{u}, \boldsymbol{u}^{\prime}$ have algebraic coefficients. See Figure 4 for a geometrical intuition of this notion.

We say that a sequence of real numbers $\left\langle x_{n}: n \in \mathbb{N}\right\rangle$ is positive if $x_{n}>0$ for all $n \in \mathbb{N}$. We moreover say that $\left\langle x_{n}: n \in \mathbb{N}\right\rangle$ is ultimately positive if there exists $N \in \mathbb{N}$ such that $x_{n}>0$ for all $n \geq N$.

Proposition 3.7. Suppose that $\tau, \tau^{\prime} \in \mathbb{R}^{d}$ are such that $\boldsymbol{\tau}^{\prime} \in$ $\operatorname{Dom}_{\mathcal{L}}(\boldsymbol{\tau})$. Then for all $\boldsymbol{u}, \boldsymbol{u}^{\prime} \in \operatorname{Ext}(U)$, if the sequence $\left\langle A^{n}\left(\boldsymbol{u}-\boldsymbol{u}^{\prime}\right), \boldsymbol{\tau}\right\rangle$ is positive (resp. ultimately positive) then there exists $\varepsilon>0$ such that the sequence $\left\langle A^{n}\left(\boldsymbol{u}-\boldsymbol{u}^{\prime}\right), \boldsymbol{\tau}+\varepsilon \boldsymbol{\tau}^{\prime}\right\rangle$ is also positive (resp. ultimately positive).

Proof. By Proposition 3.6 we have that

$$
\left\langle A^{n}\left(\boldsymbol{u}-\boldsymbol{u}^{\prime}\right), \boldsymbol{\tau}\right\rangle=\sum_{i=1}^{k} \sum_{j=0}^{d-1}\left(\begin{array}{l}
n \\
j
\end{array}\right) \lambda_{i} L_{i j}\left(\boldsymbol{u}-\boldsymbol{u}^{\prime}, \boldsymbol{\tau}\right) .
$$

Define a dominance ordering $<$ on the terms in (9), where $\left(\begin{array}{l}n \\ j\end{array}\right) \lambda_{i}^{n}<$ $\left(\begin{array}{l}n \\ \ell\end{array}\right) \lambda_{p}^{n}$ if $\lambda_{i}<\lambda_{p}$ or $\lambda_{i}=\lambda_{j}$ and $j<\ell$. Clearly $\left(\begin{array}{l}n \\ j\end{array}\right) \lambda_{i}^{n}<\left(\begin{array}{l}n \\ \ell\end{array}\right) \lambda_{p}^{n}$ implies that $\left(\begin{array}{l}n \\ j\end{array}\right) \lambda_{i}^{n}=o\left(\left(\begin{array}{l}n \\ \ell\end{array}\right) \lambda_{p}^{n}\right)$ as $n \rightarrow \infty$.

Let $\left(\begin{array}{l}n \\ j_{0}\end{array}\right) \lambda_{i_{0}}^{n}$ be the dominant term in the expansion (9) of $\left\langle A^{n}(\boldsymbol{u}-\right.$ $\left.\left.\boldsymbol{u}^{\prime}\right), \boldsymbol{\tau}\right\rangle$. Since $\boldsymbol{\tau}^{\prime} \in \operatorname{Dom}_{\mathcal{L}}(\boldsymbol{\tau})$, the expansion of $\left\langle A^{n}\left(\boldsymbol{u}-\boldsymbol{u}^{\prime}\right), \boldsymbol{\tau}^{\prime}\right\rangle$ does not have any term that strictly dominates $\left(\begin{array}{c}n \\ j_{0}\end{array}\right) \lambda^{i_{0}}$. It follows

\footnotetext{
${ }^{2}$ The eigenvalues are algebraic, being roots of the characteristic polynomial. The (gen eralized) eigenvectors are then algebraic, being solutions to linear equations with algebraic coefficients. The matrices $D$ and $P$ can then be defined in terms of eigenvalues and (generalized) eigenvectors. See [7] for more details.
}

that $\left\langle A^{n}\left(\boldsymbol{u}-\boldsymbol{u}^{\prime}\right), \boldsymbol{\tau}^{\prime}\right\rangle=O\left(\left\langle A^{n}\left(\boldsymbol{u}-\boldsymbol{u}^{\prime}\right), \boldsymbol{\tau}\right\rangle\right)$ as $n \rightarrow \infty$. In particular, there exists absolute constants $N \in \mathbb{N}$ and $K>0$ such that

$$
\left.\mid\left\langle A^{n}\left(\boldsymbol{u}-\boldsymbol{u}^{\prime}\right), \boldsymbol{\tau}\right\rangle\right) \mid \leqslant K\left\langle A^{n}\left(\boldsymbol{u}-\boldsymbol{u}^{\prime}\right), \boldsymbol{\tau}\right\rangle
$$

for all $n \geqslant N$, since the sequence $\left\langle A^{n}\left(\boldsymbol{u}-\boldsymbol{u}^{\prime}\right), \boldsymbol{\tau}\right\rangle$ is positive (resp. ultimately positive, in which case we just have to take $N$ large enough). Then for any $\epsilon>0$ and $n \geqslant N$,

$$
\begin{aligned}
\left\langle A^{n}\left(\boldsymbol{u}-\boldsymbol{u}^{\prime}\right), \boldsymbol{\tau}+\varepsilon \boldsymbol{\tau}^{\prime}\right\rangle & =\left\langle A^{n}\left(\boldsymbol{u}-\boldsymbol{u}^{\prime}\right), \boldsymbol{\tau}\right\rangle+\varepsilon\left\langle A^{n}\left(\boldsymbol{u}-\boldsymbol{u}^{\prime}\right), \boldsymbol{\tau}^{\prime}\right\rangle \\
& \geqslant(1-\epsilon K)\left\langle A^{n}\left(\boldsymbol{u}-\boldsymbol{u}^{\prime}\right), \boldsymbol{\tau}\right\rangle>0
\end{aligned}
$$

if $\epsilon<K^{-1}$. In particular, this proves that $\left\langle A^{n}\left(\boldsymbol{u}-\boldsymbol{u}^{\prime}\right), \boldsymbol{\tau}+\varepsilon \boldsymbol{\tau}^{\prime}\right\rangle$ is ultimately positive. On the other hand, for $n<N$, observe that since

$$
\left\langle A^{n}\left(\boldsymbol{u}-\boldsymbol{u}^{\prime}\right), \boldsymbol{\tau}+\varepsilon \boldsymbol{\tau}^{\prime}\right\rangle=\left\langle A^{n}\left(\boldsymbol{u}-\boldsymbol{u}^{\prime}\right), \boldsymbol{\tau}\right\rangle+\varepsilon\left\langle A^{n}\left(\boldsymbol{u}-\boldsymbol{u}^{\prime}\right), \boldsymbol{\tau}^{\prime}\right\rangle,
$$

by making $\varepsilon$ suitably small we can ensure that $\left\langle A^{n}\left(\boldsymbol{u}-\boldsymbol{u}^{\prime}\right), \boldsymbol{\tau}+\varepsilon \boldsymbol{\tau}^{\prime}\right\rangle$ is positive if $\left\langle A^{n}\left(\boldsymbol{u}-\boldsymbol{u}^{\prime}\right), \boldsymbol{\tau}\right\rangle$ is positive.

Proposition 3.8. Let $\tau, \tau^{\prime} \in \mathbb{R}^{d}$ be such that $\boldsymbol{\tau}^{\prime} \in \operatorname{Dom}_{\mathcal{L}}(\boldsymbol{\tau})$ and $\tau^{\prime} \in \operatorname{Aff}_{0}\left(\max _{\tau}\left(A^{\infty}(U)\right)\right)^{\perp}$. Then for $\varepsilon$ sufficiently small we have $\max _{\tau}\left(A^{\infty}(U)\right) \subseteq \max _{\tau+\varepsilon \tau^{\prime}}\left(A^{\infty}(U)\right)$.

Proof. Define the set of eventually $\tau$-maximizing inputs to be $U_{\boldsymbol{\tau}}^{\max }=\left\{\boldsymbol{u} \in \operatorname{Ext}(U): \forall \boldsymbol{v} \in \operatorname{Ext}(U):\left\langle A^{n}(\boldsymbol{u}-\boldsymbol{v}), \boldsymbol{\tau}\right\rangle\right.$ is identically 0 or ultimately positive $\}$.

We claim that $U_{\tau}^{\max }$ is non-empty. To see this, note that every sequence of the form $\left\langle A^{n}(\boldsymbol{u}-\boldsymbol{v}), \boldsymbol{\tau}\right\rangle$ is either identically zero or ultimately negative or ultimately positive (depending on the sign of its leading term). Thus we can define a linear preorder on the vertices of $U$ in which $\boldsymbol{u}$ greater than $\boldsymbol{v}$ if the sequence $\left\langle A^{n}(\boldsymbol{u}-\boldsymbol{v}), \boldsymbol{\tau}\right\rangle$ is ultimately non-negative. Since $U$ has a finite number of vertices, there is a maximal element under this preorder, which is then an element of $U_{\tau}^{\max }$. This establishes the claim. We further observe, that if $\sum_{i=0}^{\infty} A^{i} \boldsymbol{u}_{i} \in \max _{\boldsymbol{\tau}}\left(A^{\infty}(U)\right)$ then there exists $N \in \mathbb{N}$ such that for all $i \geq N, \boldsymbol{u}_{i} \in \operatorname{Conv}\left(U_{\tau}^{\max }\right)$, i.e., after some point the input $\boldsymbol{u}_{i}$ must be chosen in the convex hull of the set of eventually $\tau$-maximizing inputs.

We claim that for $\varepsilon>0$ small enough we have $\max _{\tau}\left(A^{i}(U)\right) \subseteq$ $\max _{\tau+\epsilon \tau^{\prime}}\left(A^{i}(U)\right)$ for $i=0, \ldots, N-1$. Indeed, $\operatorname{Aff}_{0}\left(\max _{\tau}\left(A^{i}(U)\right)\right) \subseteq$ $\mathrm{Aff}_{0}\left(\max _{\tau}\left(A^{\infty}(U)\right)\right)$ by Proposition 3.5, thus $\tau^{\prime} \in \operatorname{Aff}_{0}\left(\max _{\tau}\left(A^{i}(U)\right)\right)^{\perp}$. Let $A^{i} \boldsymbol{u} \in \max _{\boldsymbol{\tau}}\left(A^{i}(U)\right)$ and $\boldsymbol{v} \in U$, then $\left\langle A^{i}(\boldsymbol{u}-\boldsymbol{v}), \boldsymbol{\tau}\right\rangle \geqslant 0$. There are two cases to consider: if $\left\langle A^{i}(\boldsymbol{u}-\boldsymbol{v}), \boldsymbol{\tau}\right\rangle=0$ then $A^{i} \boldsymbol{v} \in$ $\max _{\boldsymbol{\tau}}\left(A^{i}(U)\right)$ thus $\left\langle A^{i}(\boldsymbol{u}-\boldsymbol{v}), \boldsymbol{\tau}+\epsilon \boldsymbol{\tau}^{\prime}\right\rangle=\epsilon\left\langle A^{i}(\boldsymbol{u}-\boldsymbol{v}), \boldsymbol{\tau}^{\prime}\right\rangle=0$. If $\left\langle A^{i}(\boldsymbol{u}-\boldsymbol{v}), \boldsymbol{\tau}\right\rangle>0$ then $\left\langle A^{i}(\boldsymbol{u}-\boldsymbol{v}), \boldsymbol{\tau}+\epsilon \boldsymbol{\tau}^{\prime}\right\rangle=\left\langle A^{i}(\boldsymbol{u}-\boldsymbol{v}), \boldsymbol{\tau}+\epsilon \boldsymbol{\tau}^{\prime}\right\rangle \geqslant 0$ for small enough $\epsilon$.

We claim that $U_{\tau}^{\max } \subseteq U_{\tau+\varepsilon \tau^{\prime}}^{\max }$ for $\varepsilon$ sufficiently small. Aside we have seen that for $\varepsilon$ small enough we have $\max _{\tau}\left(A^{i}(U)\right) \subseteq$ $\max _{\tau+\epsilon \tau^{\prime}}\left(A^{i}(U)\right)$ for $i=0, \ldots, N-1$. It follows that for $\varepsilon$ sufficiently small we have $\max _{\tau}\left(A^{\infty}(U)\right) \subseteq \max _{\tau+\varepsilon \tau^{\prime}}\left(A^{\infty}(U)\right)$, as we wanted to prove.

It remains to prove the claim. To this end, consider $\boldsymbol{u} \in U_{\boldsymbol{\tau}}^{\max }$ and $\boldsymbol{v} \in \operatorname{Ext}(U)$. If the sequence $\left\langle A^{n}(\boldsymbol{u}-\boldsymbol{v}), \boldsymbol{\tau}\right\rangle$ is identically zero then also $v \in U_{\tau}^{\max }$. Since $\boldsymbol{\tau}^{\prime} \in \operatorname{Aff}_{0}\left(\max _{\boldsymbol{\tau}}\left(A^{i}(U)\right)\right)^{\perp}$ for all $i \in \mathbb{N}$ it follows that $\left\langle A^{n}(\boldsymbol{u}-\boldsymbol{v}), \boldsymbol{\tau}+\varepsilon \boldsymbol{\tau}^{\prime}\right\rangle$ is also identically zero. On the other hand, if $\left\langle A^{n}(\boldsymbol{u}-\boldsymbol{v}), \boldsymbol{\tau}\right\rangle$ is ultimately positive then for $\epsilon$ small enough $\left\langle A^{n}(\boldsymbol{u}-\boldsymbol{v}), \boldsymbol{\tau}+\varepsilon \boldsymbol{\tau}^{\prime}\right\rangle$ is also ultimately positive by Proposition 3.7. This proves the claim and concludes the proof. 


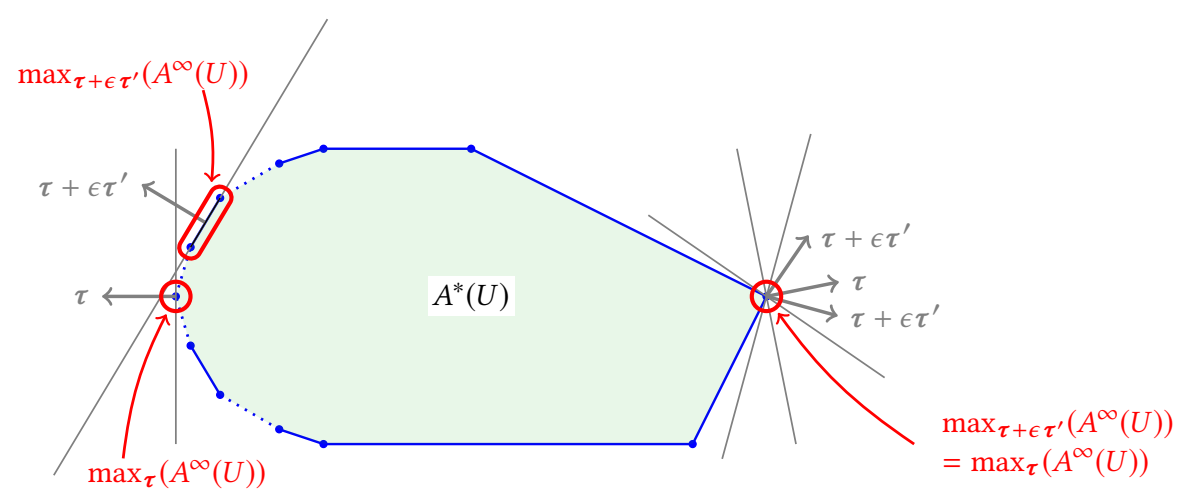

Figure 4: The set $\operatorname{Dom}_{\mathcal{L}}(\tau)$ contains directions $\tau^{\prime}$ such that the sequence $\left\langle A^{n}\left(u-u^{\prime}\right), \tau^{\prime}\right\rangle$ does not asymptotically dominate $\left\langle A^{n}\left(u-u^{\prime}\right), \tau\right\rangle$ in absolute value (for all $u, u^{\prime} \in U$ ). Intuitively, eventual $\tau$ maximizers in $U$ are also eventual $\tau+\varepsilon \tau^{\prime}$ maximizers for $\varepsilon$ small enough. Thus the face of $A^{\infty}(U)$ in direction $\tau+\epsilon \tau^{\prime}$ is the same as the one in direction $\tau$ for small $\epsilon$ (see Proposition 3.8), as illustrated on the right. When $\tau^{\prime}$ asymptotically dominates $\tau$, any perturbation of $\tau$ will result in a different face, as illustrated on the left. In particular this can happen when there is an infinite number of faces converging to a single point (as in Figure 3 ).

Proposition 3.9. If $\tau$ is an extremal vector in $\operatorname{Cone}\left(A^{\infty}(U), Q\right)$ then

$$
\operatorname{Dom}_{\mathcal{L}}(\tau) \cap \operatorname{Aff}_{0}\left(\max _{\tau}\left(A^{\infty}(U)\right) \cup \min _{\tau}(Q)\right)^{\perp}=\operatorname{Span}(\tau) .
$$

Proof. We first show the right-to-left inclusion, for which it suffices to show that $\tau$ lies in the left-hand side. It is clear that $\tau \in \operatorname{Aff}_{0}\left(\max _{\boldsymbol{\tau}}\left(A^{\infty}(U)\right)\right)^{\perp}$ and $\boldsymbol{\tau} \in \operatorname{Aff}_{0}\left(\min _{\tau}(Q)\right)^{\perp}$ (e.g., if $\boldsymbol{u}, \boldsymbol{v} \in$ $\min _{\boldsymbol{\tau}}(Q)$ then we have $\langle\boldsymbol{u}, \boldsymbol{\tau}\rangle=\langle\boldsymbol{v}, \boldsymbol{\tau}\rangle$ and hence $\langle\boldsymbol{u}-\boldsymbol{v}, \boldsymbol{\tau}\rangle=0$ ). Now consider $\boldsymbol{u} \in \max _{\boldsymbol{\tau}}\left(A^{\infty}(U)\right)$ and $\boldsymbol{v} \in \min _{\tau}(Q)$. By definition we have $\langle\boldsymbol{u}, \boldsymbol{\tau}\rangle \leq\langle\boldsymbol{v}, \boldsymbol{\tau}\rangle$. But if $\langle\boldsymbol{u}, \boldsymbol{\tau}\rangle\langle\langle\boldsymbol{v}, \boldsymbol{\tau}\rangle$, then since both $A^{\infty}(U)$ and $Q$ are bounded we have that $\tau$ lies in the interior of Cone $\left(A^{\infty}(U), Q\right)$ contradicting the assumption that $\tau$ is extremal. Thus we must have $\langle\boldsymbol{u}, \boldsymbol{\tau}\rangle=\langle\boldsymbol{v}, \boldsymbol{\tau}\rangle$ and hence $\langle\boldsymbol{u}-\boldsymbol{v}, \boldsymbol{\tau}\rangle=0$. We conclude that $\tau \in \mathrm{Aff}_{0}\left(\max _{\tau}\left(A^{\infty}(U)\right) \cup \min _{\tau}(Q)\right)^{\perp}$. This completes the proof of the right-to-left inclusion.

For the left-to-right inclusion, consider a vector $\tau^{\prime}$ contained in the left-hand side of (10). We claim that for suitably small $\varepsilon \in \mathbb{R}$, both $\boldsymbol{\tau}+\varepsilon \boldsymbol{\tau}^{\prime}$ and $\boldsymbol{\tau}-\varepsilon \boldsymbol{\tau}^{\prime}$ lie in $\operatorname{Cone}\left(A^{\infty}(U), Q\right)$. Since $\boldsymbol{\tau}$ is extremal in Cone $\left(A^{\infty}(U), Q\right)$ we conclude that $\tau^{\prime} \in \operatorname{Span}(\tau)$.

It remains to prove the claim. To this end, notice that for $\boldsymbol{u} \in$ $\max _{\boldsymbol{\tau}}\left(A^{\infty}(U)\right), \boldsymbol{v} \in \min _{\boldsymbol{\tau}}(Q)$, and $\varepsilon \in \mathbb{R}$, we have $\langle\boldsymbol{u}-\boldsymbol{v}, \boldsymbol{\tau}+$ $\left.\varepsilon \boldsymbol{\tau}^{\prime}\right\rangle=\langle\boldsymbol{u}-\boldsymbol{v}, \boldsymbol{\tau}\rangle=0$. Moreover by Proposition 3.8, for $\varepsilon$ suitably small we have $\max _{\tau}\left(A^{\infty}(U)\right) \subseteq \max _{\tau+\varepsilon \tau^{\prime}}\left(A^{\infty}(U)\right)$. Similar but simpler reasoning to the proof of Proposition 3.8 also yields that $\min _{\tau}(Q) \subseteq \min _{\tau+\varepsilon \tau^{\prime}}(Q)$ for $\varepsilon$ small enough. It follows that $\left\langle\boldsymbol{u}, \boldsymbol{\tau}+\varepsilon \boldsymbol{\tau}^{\prime}\right\rangle=\left\langle\boldsymbol{v}, \boldsymbol{\tau}+\varepsilon \boldsymbol{\tau}^{\prime}\right\rangle$ for all $\boldsymbol{u} \in \max _{\boldsymbol{\tau}+\varepsilon \boldsymbol{\tau}^{\prime}}\left(A^{\infty}(U)\right)$ and all $\boldsymbol{v} \in \min _{\tau+\varepsilon \tau^{\prime}}(Q)$. Thus $\tau+\varepsilon \tau^{\prime}$ separates $A^{*}(U)$ from $Q$, establishing the claim.

We can now show our separation lemma.

Proof of Lemma 3.4. Recall that $\operatorname{Cone}\left(A^{\infty}(U), Q\right)$ is a closed pointed cone. It follows that it has an extremal vector $\tau$. By Proposition 3.5 and Proposition 3.9 we can assume that all entries of $\tau$ are algebraic. Indeed we have already noted that $\operatorname{Dom}_{\mathcal{L}}(\tau)$ and $\operatorname{Aff}_{0}\left(\min _{\tau} Q\right)$ ) have a basis of rational vectors. We conclude that if there is a separator of $A^{*}(U)$ and $Q$ then there is an algebraic separator.

THEOREM 3.10. The reachability problem for simple LTI systems is decidable.

Proof. As we have noted above, it suffices to give a semi-decision procedure to show that a target $Q \subseteq \mathbb{R}^{d}$ is not reachable in a given LTI system $\mathcal{L}=(A, U)$. To show this we enumerate all vectors $\tau \in \mathbb{R}^{d}$ with algebraic entries and determine whether $\tau$ separates $A^{\infty}(U)$ from $Q$, that is, whether

$$
\left\{\langle\boldsymbol{u}, \boldsymbol{\tau}\rangle: \boldsymbol{u} \in A^{\infty}(U)\right\} \leq \min \{\langle\boldsymbol{v}, \boldsymbol{\tau}\rangle: \boldsymbol{v} \in Q\} .
$$

It is straightforward to calculate right-hand side in (11). To compute left-hand side the idea is to find some eventually $\tau$-maximising input $\boldsymbol{u} \in \operatorname{Ext}(U)$ and corresponding threshold $N \in \mathbb{N}$ such that for all $\boldsymbol{v} \in \operatorname{Ext}(U)$ and all $i \geq N$ we have $\left\langle A^{i} \boldsymbol{u}, \boldsymbol{\tau}\right\rangle \geq\left\langle A^{i} \boldsymbol{v}, \boldsymbol{\tau}\right\rangle$. Given $\boldsymbol{u}$ and $N$, we obtain an element of $\max _{\tau}\left(A^{\infty}(U)\right)$ as

$$
\sum_{i=0}^{N-1} A^{i} \boldsymbol{u}_{i}+A^{N} \sum_{i=0}^{\infty} A^{i} \boldsymbol{u}=\sum_{i=0}^{N-1} A^{i} \boldsymbol{u}_{i}+A^{N}(I-A)^{-1} \boldsymbol{u}
$$

where $\boldsymbol{u}_{i} \in \max _{\tau}\left(A^{i}(U)\right)$ for $i=0, \ldots, N-1$.

It remains to find such a $\boldsymbol{u}$ and $N$. For this we consider each sequence of the form $\left\langle A^{i}(\boldsymbol{v}-\boldsymbol{w}), \boldsymbol{\tau}\right\rangle$ for $\boldsymbol{v}, \boldsymbol{w} \in \operatorname{Ext}(U)$. Each such sequence is either identically zero, ultimately positive, or ultimately negative. Moreover by examining the dominant term of the sequence we can decide which of these eventualities is the case and, in case of an ultimately positive sequence, compute the index from which the sequence becomes positive. Clearly this is enough to determine which of the extremal points of $U$ is an ultimate $\tau$-maximiser and to determine the corresponding threshold $N$.

\section{CONCLUSION}

Our main result showed decidability of the LTI Reachability Problem for so-called simple LTI systems. The most restrictive condition in the notion of a simple LTI system (see Section 3) is that 
some power of the transition matrix have real spectrum. This assumption was crucial in proving that every unreachable point is separated from the set of reachable points by a hyperplane with algebraic coefficients. To illustrate the difficulty with eliminating or weakening this assumption, consider the following LTI system in which the transition matrix performs a counter clockwise rotation in the plane by angle $\theta$ :

$$
A=\frac{1}{2}\left(\begin{array}{cc}
\cos \theta & -\sin \theta \\
\sin \theta & \cos \theta
\end{array}\right), \quad U=[0,1] \times\{0\} .
$$

Considering the direction $\tau=(1,0)$, it is not hard to see that the furthest we can go in direction $\tau$ is

$$
\max _{x \in A^{\infty}(U)}\langle x, \tau\rangle=\sum_{n=0}^{\infty} \max \left(0,2^{-n} \cos (n \theta)\right) .
$$

Now $A$ has eigenvalues $\lambda=\frac{1}{2} e^{i \theta}$ and $\bar{\lambda}=\frac{1}{2} e^{-i \theta}$. If $A$ is simple then a positive power of $\lambda$ must be real, in other words $\theta$ is a rational multiple of $\pi$. In this case the sign of $\cos (n \theta)$ is periodic and we can find an explicit expression for the sum above and thereby show that it is an algebraic number. However if $\theta$ is an irrational multiple of $\pi$ and the sign of $\cos (n \theta)$ is hard to analyse. In particular, we do not know if the resulting sum is an algebraic number.

\section{REFERENCES}

[1] A. Jamak. Stabilization of discrete-time systems with bounded control inputs. Master's thesis, University of Waterloo, 2000. URL: http://hdl.handle.net/10012/765.

[2] S. Akshay, T. Antonopoulos, J. Ouaknine, and J. Worrell. Reachability problems for markov chains. Information Processing Letters, 115(2):155 - 158, 2015.

[3] E. Asarin, O. Maler, and A. Pnueli. Reachability analysis of dynamical systems having piecewise-constant derivatives. Theor. Comput. Sci., 138(1):35-65, 1995.

[4] V. Blondel and J. Tsitsiklis. Complexity of stability and controllability of elementary hybrid systems. Automatica, 35(3):479-489, 1999.

[5] V. Blondel and J. Tsitsiklis. Overview of complexity and decidability results for three classes of elementary nonlinear systems. In Yutaka Yamamoto and Shinji Hara, editors, Learning, control and hybrid systems, pages 46-58, London, 1999. Springer.

[6] V. Blondel and J. Tsitsiklis. A survey of computational complexity results in systems and control. Automatica, 36(9):1249-1274, 2000.

[7] Jin-Yi Cai. Computing Jordan normal forms exactly for commuting matrices in polynomial time. Technical report, SUNY at Buffalo, 2000.

[8] D. Cattaruzza, A. Abate, P. Schrammel, and D. Kroening. Unbounded-time analysis of guarded LTI systems with inputs by abstract acceleration. In Static Analysis - 22nd International Symposium, SAS, volume 9291 of Lecture Notes in Computer Science, pages 312-331. Springer, 2015.

[9] P. Cook. On the behaviour of dynamical systems subject to bounded disturbances. International fournal of Systems Science, 11(2):159-170, 1980.

[10] Nathanaël Fijalkow, Joël Ouaknine, Amaury Pouly, João Sousa Pinto, and James Worrell. On the decidability of reachability in linear time-invariant systems. CoRR, abs/1802.06575, 2018. URL: http://arxiv.org/abs/1802.06575, arXiv: 1802.06575

[11] A. Girard and C. Le Guernic. Efficient reachability analysis for linear systems using support functions. IFAC Proceedings Volumes, 41(2):8966 - 8971, 2008. 17th IFAC World Congress.

[12] A. Girard, C. Le Guernic, and O. Maler. Efficient computation of reachable sets of linear time-invariant systems with inputs. In Hybrid Systems: Computation and Control, 9th International Workshop, HSCC, volume 3927 of Lecture Notes in Computer Science, pages 257-271. Springer, 2006.

[13] W. J. Grantham and T. L. Vincent. A controllability minimum principle. fournal of Optimization Theory and Applications, 17(1):93-114, Oct 1975.

[14] V. Halava, T. Harju, M. Hirvensalo, and J. Karhumäki. Skolem's problem - on the border between decidability and undecidability. Technical Report 683, Turku Centre for Computer Science, 2005.

[15] M. A. Harrison. Introduction to Formal Language Theory. Addison-Wesley Longman Publishing Co., Inc., Boston, MA, USA, 1st edition, 1978

[16] W. P. M. H. Heemels and M. K. Camlibel. Null controllability of discrete-time linear systems with input and state constraints. In 2008 47th IEEE Conference on Decision and Control, pages 3487-3492, 2008.
[17] T. Hu and D. Miller. Null controllable region of LTI discrete-time systems with input saturation. Automatica, 38(11):2009 - 2013, 2002.

[18] T. Hu, D. Miller, and L. Qiu. An explicit description of null controllable regions of linear systems with saturating actuators. Systems \& Control Letters, 47(1):65 $-78,2002$.

[19] T. Hu and L. Qiu. Controllable regions of linear systems with bounded inputs. Systems and Control Letters, 33(1):55-61, 1998.

[20] R. Kannan and R. J. Lipton. Polynomial-time algorithm for the orbit problem. F. ACM, 33(4):808-821, August 1986.

[21] Shahab Kaynama and Meeko M. K. Oishi. Overapproximating the reachable sets of lti systems through a similarity transformation. Proceedings of the 2010 American Control Conference, pages 1874-1879, 2010.

[22] I. Kirat and I. Kocyigit. On the Convex Hulls of Self-Affine Fractals. ArXiv eprints, April 2015. arXiv: 1504.07396.

[23] P. Koiran, M. Cosnard, and M. Garzon. Computability with low-dimensional dynamical systems. Theor. Comput. Sci., 132(2):113-128, 1994.

[24] Gerardo Lafferriere, George J. Pappas, and Shankar Sastry. O-minimal hybrid systems. MCSS, 13(1):1-21, 2000 .

[25] M. Mignotte, T. Shorey, and R. Tijdeman. The distance between terms of an algebraic recurrence sequence. F. für die reine und angewandte Math., 349, 1984.

[26] J. Ouaknine and J. Worrell. Positivity problems for low-order linear recurrence sequences. In Proceedings of the Twenty-Fifth Annual ACM-SIAM Symposium on Discrete Algorithms, SODA 2014, pages 366-379, 2014.

[27] G. Rozenberg and A. Salomaa. Cornerstones of Undecidability. Prentice Hall, 1994.

[28] A. Salomaa and M. Soittola. Automata-Theoretic Aspects of Formal Power Series. Texts and Monographs in Computer Science. Springer, 1978.

[29] W. E. Schmitendorf and B. R. Barmish. Null controllability of linear systems with constrained controls. SIAM fournal on Control and Optimization, 18(4):327-345, 1980.

[30] O. Shakernia, S. Sastry, and G. J. Pappas. Decidable controller synthesis for classes of linear systems. In Hybrid Systems: Computation and Control, Third International Workshop, HSCC, volume 1790 of Lecture Notes in Computer Science, pages 407-420. Springer, 2000.

[31] H. Siegelmann and E. Sontag. On the computational power of neural nets. $\mathcal{F}$. Comput. Syst. Sci., 50(1):132-150, 1995.

[32] E. Sontag. An algebraic approach to bounded controllability of linear systems. International fournal of Control, 39(1):181-188, 1984.

[33] D. Summers, Z. Wu, and C. Sabin. State estimation of linear dynamical systems under bounded control. Fournal of Optimization Theory and Applications, 72:299818, 1992.

[34] T. Tao. Structure and randomness: pages from year one of a mathematical blog. American Mathematical Society, 2008.

[35] R. van Til and W.E. Schmitendorf. Constrained controllability of discrete-time systems. International fournal of Control, 43(3):941-956, 1986.

[36] J. Vass. On the Exact Convex Hull of IFS Fractals. ArXiv e-prints, February 2015. arXiv: 1502.03788.

[37] N. K. Vereshchagin. The problem of appearance of a zero in a linear recurrence sequence (in Russian). Mat. Zametki, 38(2), 1985.

[38] M. Zhao. On Controllable Abundance Of Saturated-input Linear Discrete Systems. ArXiv e-prints, May 2017. arXiv: 1705.08064. 


\section{A UNDECIDABILITY FOR INVERTIBLE MATRIX PROBLEMS}

Given $k+1$ invertible matrices $A_{1}, \ldots, A_{k}, C \in \mathbb{Q}^{d \times d}$, the generalized matrix powering problem for invertible matrices consists in deciding whether there exist $n_{1}, \ldots, n_{k} \in \mathbb{Z} \backslash\{0\}$ such that

$$
\prod_{i=1}^{k} A_{i}^{n_{i}}=C
$$

The following results are folklore but we could not find any proof of them in the literature.

Theorem A.1. The generalized matrix powering problem for invertible matrices is undecidable.

Proof. We will show this result by reducing from Hilbert's Tenth Problem. Given a polynomial $p \in \mathbb{Z}\left[n_{1}, \ldots, n_{k}\right]$, it is easy to express $p\left(n_{1}, \ldots, n_{k}\right)$ as a conjunction of relations of the following form (noting that we may need to introduce new variables):

- $z=k$, where $k \in \mathbb{Z}$

- $z=x+y$

- $z=x y$.

We start by showing how to encode each of these as an instance of the generalized matrix powering problem for invertible matrices. Firstly, note that

$$
z=k \Leftrightarrow\left(\begin{array}{ll}
1 & 1 \\
0 & 1
\end{array}\right)^{z}=\left(\begin{array}{ll}
1 & k \\
0 & 1
\end{array}\right)
$$

Secondly, note that

$$
z=x+y \Leftrightarrow\left(\begin{array}{ll}
1 & 1 \\
0 & 1
\end{array}\right)^{x}\left(\begin{array}{ll}
1 & 1 \\
0 & 1
\end{array}\right)^{y}\left(\begin{array}{cc}
1 & -1 \\
0 & 1
\end{array}\right)^{z}=\left(\begin{array}{ll}
1 & 0 \\
0 & 1
\end{array}\right)
$$

Thirdly, note that

$$
z=x y \Leftrightarrow \exists x^{\prime}, y^{\prime} \in \mathbb{Z},\left(\begin{array}{lll}
1 & 0 & 0 \\
0 & 1 & 0 \\
0 & 0 & 1
\end{array}\right)=\left(\begin{array}{ccc}
1 & x-x^{\prime} & z-x y \\
0 & 1 & y-y^{\prime} \\
0 & 0 & 1
\end{array}\right)
$$

and that the latter matrix is just equal to

$$
\left(\begin{array}{ccc}
1 & 0 & -1 \\
0 & 1 & 0 \\
0 & 0 & 1
\end{array}\right)^{z}\left(\begin{array}{ccc}
1 & 0 & 0 \\
0 & 1 & -1 \\
0 & 0 & 1
\end{array}\right)^{y^{\prime}}\left(\begin{array}{ccc}
1 & 1 & 0 \\
0 & 1 & 0 \\
0 & 0 & 1
\end{array}\right)^{x}\left(\begin{array}{ccc}
1 & 0 & 0 \\
0 & 1 & 1 \\
0 & 0 & 1
\end{array}\right)^{y}\left(\begin{array}{ccc}
1 & -1 & 0 \\
0 & 1 & 0 \\
0 & 0 & 1
\end{array}\right)^{x^{\prime}}
$$

Finally, conjunction can be achieved by making use of separate matrix blocks:

$$
\prod_{i=1}^{k} A_{i}^{n_{i}}=C \wedge \prod_{i=1}^{k} B_{i}^{n_{i}}=D \Leftrightarrow \prod_{i=1}^{k}\left(\begin{array}{cc}
A_{i} & 0 \\
0 & B_{i}
\end{array}\right)^{n_{i}}=\left(\begin{array}{ll}
C & 0 \\
0 & D
\end{array}\right) .
$$

Definition A.2. Given invertible matrices $A_{1}, \ldots, A_{k} \in \mathbb{Q}^{d \times d}$ and two non-zero vectors $\boldsymbol{x}, \boldsymbol{y} \in \mathbb{Q}^{d}$, the vector reachability problem for invertible matrices consists in deciding whether there exist $n_{1}, \ldots, n_{k} \in \mathbb{Z} \backslash\{0\}$ such that

$$
\prod_{i=1}^{k} A_{i}^{n_{i}} \boldsymbol{x}=\boldsymbol{y}
$$

THEOREM A.3. The vector reachability problem for invertible matrices is undecidable.
Proof. This can be shown by reduction from the generalised matrix powering problem for invertible matrices. In particular, given invertible matrices $A_{1}, \ldots, A_{k}, B \in \mathbb{Q}^{d \times d}$, letting $\boldsymbol{b}_{1}, \ldots, \boldsymbol{b}_{d}$ denote the columns of $B$, and letting $\boldsymbol{e}_{1}, \ldots, \boldsymbol{e}_{d}$ denote the canonical basis of $\mathbb{R}^{d}$, the result follows from the fact that

$$
\prod_{i=1}^{k} A_{i}^{n_{i}}=B \Leftrightarrow \prod_{i=1}^{k}\left(\begin{array}{ccc}
A_{i} & \cdots & 0 \\
\vdots & \ddots & \vdots \\
0 & \cdots & A_{i}
\end{array}\right)^{n_{i}}\left(\begin{array}{c}
\boldsymbol{e}_{1} \\
\vdots \\
\boldsymbol{e}_{d}
\end{array}\right)=\left(\begin{array}{c}
\boldsymbol{b}_{1} \\
\vdots \\
\boldsymbol{b}_{d}
\end{array}\right) .
$$

